\title{
De un vistazo
}

\section{Estrategias diagnósticas en pacientes con sospecha de trombosis venosa profunda}

Estudio aleatorizado de estrategias diagnósticas después de ecografía venosa proximal normal en la sospecha de trombosis venosa profunda: dímero-d comparado con la repetición de la ecografía. Kearon c. y col. Ann Intern Med. 2005;142:490-6

La compresión venosa por ecografía es el test diagnóstico de elección para la sospecha de trombosis venosa profunda (TVP). Si se confirma la TVP con la ecografía, es necesario la anticoagulación del paciente, pero el problema es en caso que el estudio sea normal, teniendo en cuenta que la ecografía es menos precisa para evaluar venas profundas que superficiales y que se puede producir la TVP hasta una semana después de la sospecha diagnóstica, lo conveniente es anticoagular en forma preventiva y repetir el estudio en una semana. Esto aumenta los costos de los estudios y crea ansiedad e incertidumbre en los pacientes, por lo que se buscó un método para reducir la necesidad de pedir una nueva ecografía en estos pacientes. El dímero-D es una molécula circulante que aumenta cuando se lisa un trombo por el sistema fibrinolítico, y se eleva en pacientes con TVP. La presencia de un valor normal alejaría el diagnóstico. Varios investigadores mostraron que un resultado de aglutinación eritrocitaria de dímero-D negativo (SimpliRED, AGEN Biomedical Ltd, Australia) es válido para excluir TVP y tromboembolismo pulmonar.

Este estudio compara dos estrategias diagnósticas usadas para el manejo de pacientes con sospecha de TVP y resultado normal en la ecografía inicial 1) la combinación de dímero-D con la ecografía inicial, y si es normal obviar la necesidad de repetir la ecografía en una semana (y si es anormal realizar venografía); y 2) la repetición rutinaria de la ecografía a una semana. Se realizo un ensayo aleatorizado en 810 pacientes seguidos por seis meses con sospecha de TVP y se los dividió en dos grupos: dímero-D 408 pacientes y control rutinario 402 pacientes. En el grupo dímero-D se diagnosticó inicialmente a 19 pacientes contra 3 del grupo repetición de ecografía. De los restantes, durante el seguimiento a 6 meses 8 pacientes del grupo dímero-D desarrollaron TVP contra 5 del otro grupo. El estudio concluye que en pacientes ambulatorios con sospecha de TVP y ecografía inicial negativa el uso de dímero-D para decidir la estrategia (no realizar la ecografía a la semana de control si el dímero-D es negativo y hacer una venografía en aquellos con dímero-D positivo) fue similarmente seguro que la estrategia ecográfica.

\section{Sirolimus vs. Paclitaxel}

Stent recubiertos con sirolimus vs. paclitaxel en pacientes con enfermedad coronaria. Meta-Análisis de Estudios Aleatorizados. Kastrati A y col. JAMA, 2005; 294:819- 25

Si bien la colocación de stents en pacientes que requieren intervenciones coronarias percutáneas mejora los resultados, los stent "sin drogas" están asociados con un incremento del riesgo de reestenosis. Los stents "con drogas", que tienen la capacidad de reducir la hiperplasia neointimal por vía local de liberación de agentes antiproliferativos, han emergido como una solución efectiva al problema de las reestenosis.

Muchos ensayos clínicos así como revisiones sistemáticas confirman esta mayor efectividad contra los stent metálicos "sin drogas". Pero son pocos los trabajos que comparan las dos drogas más habituales que los recubren (sirolimus o paclitaxel), siendo estas las más usadas en EE.UU., casi en un $85 \%$ de los stent "con drogas" colocados. El motivo de este meta-análisis es evaluar la eficacia y seguridad de los stents con sirolimus vs. aquellos con paclitaxel en pacientes con enfermedad coronaria (EC).

Se evaluaron seis ensayos aleatorizados, que comparaban ambos stents en 3.669 pacientes con EC. Tres de estos estudios tenían como punto final a evaluar la reestenosis por angiografía. Otros dos tenían además como punto final la mortalidad, el infarto de miocardio (IAM) y la revascularización, y en el último estudio lo que se evaluó fue pérdida de la luz arterial en el seguimiento angiográfico. Todos tuvieron un seguimiento mínimo de seis meses. La revascularización de la lesión índice fue menor en el grupo sirolimus que en el paclitaxel (5.1\% vs. 7.8\%; OR 0,64; IC95 0,49 a 0,84). Los pacientes que recibieron stents con sirolimus también tuvieron un menor riesgo de reestenosis (9,3 vs. 13,1\%; OR 0,68; IC95 0,55 a 0,86). Esto se debería a una mayor reducción de la perdida tardía del lumen debido al sirolimus evidenciado en las angiografías de control. No se observaron diferencias significativas entre las dos drogas en el riesgo de IAM y muerte (4,9\% sirolimus vs. 5,8\% paclitaxel, $p=N S$ ). El estudio concluye que los stents recubiertos de sirulimus tuvieron menor riesgo de revascularización de lesiones índices y restenosis que los de paclitaxel, sin encontrar diferencias en eventos clínicos como muerte, IAM o trombosis del stent.

Sergio A. Boero [ Médico del Servicio de Clínica Medica, Hospital "Felipe Glasman" Bahia Blanca. ] 